\title{
Combined Method of Near Field Acoustic Holography and Focused Beamforming for Noise Source Identification in Enclosed Space
}

\author{
Zhang Lan-Yue, Wang Jia, Yang De-Sen, Shi Jie, Shi Sheng-Guo and Zhu Zhong-Rui \\ College of Underwater Acoustic Engineering, Harbin Engineering University, Harbin 150001, China, \\ Science and Technology on Underwater Acoustic Laboratory, Harbin Engineering University, Harbin 150001, \\ China.
}

(Received 12 August 2016; accepted 21 April 2017)

Noise control of vehicle cabins has been given high consideration owing to the increasing demands for comfort of ship vehicles. Noise source identification is the precondition of noise control. Conventional methods of noise source identification are mainly applied in free fields. The performance of noise source identification methods will be seriously decreased in enclosed space due to the effect of sound reflection. Noise source identification in enclosed space based on double cylindrical array is researched in this paper. The effect of reflection sound is removed by using the sound field separation technique. The combined method of near field acoustic holography and focused beamforming for noise source identification is proposed to identify noise sources having high and low frequencies. The performance of the proposed method is studied by simulation, and the experiment is carried out in the submarine cabin model to verify the feasibility of the proposed method. The researching results shows that the effect of reflection sound can be removed using sound field separation technique based on double cylindrical array, and the high-resolution identification for high and low frequency signals can be obtained using the combined method of near field acoustic holography and focused beamforming.

\section{INTRODUCTION}

Noise source identification is the key step for noise control. Conventional methods of noise source identification include near field acoustic holography and focused beamforming, but the conventional methods are usually used in the free field. ${ }^{1-6}$ In the enclosed space, such as vehicle cabin, the performance of noise source identification will be seriously decreased because of sound reflection of the wall and ceiling. ${ }^{7,8}$

Noise source identification in enclosed space is researched in recent years. In 1956, Pachner first proposed the idea of sound field separation technique. ${ }^{9}$ In 1980 , the theory of sound field separation based on spherical wave superposition method was established by Weinreich. ${ }^{10}$ In 1993, sound field was reconstructed using sound field separation technique by Hallman and Bolton. ${ }^{11-13}$ In 1995, the equation of sound field separation was given by Williams by summarizing the research results of formers. ${ }^{14}$ In 1996, inversion of reflection coefficients of arbitrary incident angle based on plane sound field separation was given by H. E. Yuan-An. ${ }^{15}$ In 2000, the noise source was located in cabin using BEM near field acoustic holography by Williams. ${ }^{16}$ In 2003, sound field separation technique was researched to extend the application range using double plane array to obtain sound pressure by Yu Fei. ${ }^{17,18}$ In 2006, the method of extended SONAH was used to locate the noise source in vehicle cabin by Hald. ${ }^{19}$ In 2007, spherical wave superposition was applied to measure the directivity and frequency response of low frequency sound equipment in a small room. ${ }^{20}$ In 2008, the sound field separation technique based on equivalent sources was studied to reconstruct the sound field in bounded space by Bi C. X. ${ }^{21}$ In 2012, spherical wave superposition method was used based on a double small half spherical array to identify the noise source in a car by Melon. ${ }^{22}$

For solving the problem of noise source identification for high and low frequency signals in an enclosed space, combined method for noise source identification is researched based on double cylindrical array in this paper. The sound field separation technique is studied to remove the effect of reflection sound coming from the walls and ceiling. The combined method of near field acoustic holography and focused beamforming are proposed to identify the location of noise source. Near field acoustic holography is used for low frequency noise source location, and focused beamforming is used for high frequency noise source location. The high resolution of noise source identification for low and high frequency signals in an enclosed space is obtained by using the proposed method.

\section{THEORETICAL ANALYSIS}

\subsection{Overview of Noise Source Identification in Enclosed Space}

The sound field in enclosed space is comprised of direct and reflected sound because of the refection of walls and ceiling. As shown in Fig. 1, noise source is in the interior of the double cylindrical array. The direct radiation sound goes out from the interior of the double cylindrical array, and the reflection sound reflected by the walls and ceiling comes in from the exterior of the double cylindrical array. The information of the sound field is acquired by the double cylindrical array. The direct and reflected sounds are separated by using the sound field separation technique. The noise sources are identified using near field acoustic holography and focused beamforming. 
The double cylindrical array

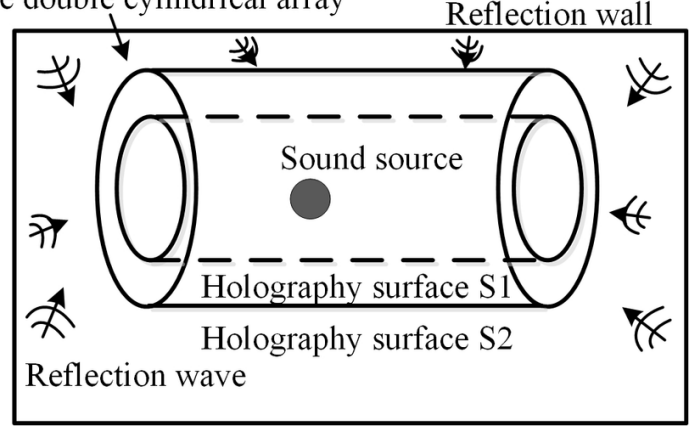

Figure 1. Sketch map of noise source identification in enclosed space using double cylindrical array.

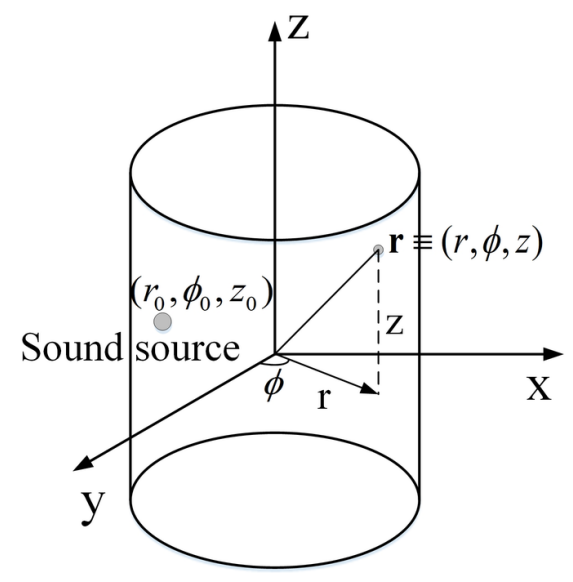

Figure 2. Cylindrical coordinate system.

\subsection{Measurement Data Model of Cylindrical Array}

The data model is given to take a single cylindrical array as example. The height of the cylindrical array is $Z$, the radius is $r_{H}$, and cylindrical coordinates $(r, \phi, z)$ system is established, which is shown in Fig. 2. The elements are uniformly distributed along the circumference direction and axis direction. The interval of elements in circumference direction is $\Delta \phi$ and the serial number is $n(n=1, \cdots, N)$, and the number of circumference elements is $N=[2 \pi / \Delta \phi]_{i n t}$, where $[\cdot]_{\text {int }}$ represents round number. The interval of elements in axis direction is $\Delta z$, the serial number is $m(m=1, \cdots, M)$, and the number of axis direction elements is $M=[Z / \Delta z]_{\text {int }}$.

The relation between cylindrical coordinates and Cartesian coordinates is $x=r \cos \phi, y=r \sin \phi, z=z$. The point sound source is in $\left(r_{0}, \phi_{0}, z_{0}\right)$ and the corresponding Cartesian coordinates are $\left(x_{0}, y_{0}, z_{0}\right)$, as shown in Fig. 2. The expression of sound pressure is given as:

$$
p(r, t)=\frac{P_{0}}{r} \mathrm{e}^{-\mathrm{i}(\omega t-k r)} ;
$$

where $P_{0}$ is the amplitude of sound pressure with distance of one meter to the sound source, $r$ is the distance of observation point to the sound source, $\omega$ is the angular frequency, $k$ is the wave number and $t$ is the observation time. The coordinates of the element with serial number $(n, m)$ are $\left(r_{n m}, \phi_{n m}, z_{n m}\right)$ and the corresponding Cartesian coordinates are $\left(x_{n m}, y_{n m}, z_{n m}\right)$. The output of element with serial num- ber $(n, m)$ is:

$$
p\left(r_{n m}, t\right)=P_{0} \frac{1}{r_{n m}} \mathrm{e}^{-\mathrm{i}\left(\omega t-k r_{n m}\right)} ;
$$

where $r_{n m}=\sqrt{\left(x_{n m}-x_{0}\right)^{2}+\left(y_{n m}-y_{0}\right)^{2}+\left(z_{n m}-z_{0}\right)^{2}}$ is the distance of the $(n, m)$ element to sound $\mathrm{nm} \mathrm{nm} 0 \mathrm{~nm} 0$ nm 0source. There are $N \times M$ microphones to compose the cylindrical array. The complex sound pressure data $\boldsymbol{p}$ of the cylindrical array is:

$$
\begin{aligned}
& \boldsymbol{p}(r, t)=\left[p_{11}\left(r_{11}, t\right) \cdots p_{n m}\left(r_{n m}, t\right) \cdots p_{N M}\left(r_{N M}, t\right)\right] \\
& =P_{0} \mathrm{e}^{-\mathrm{i} \omega t}\left[\frac{1}{r_{11}} \mathrm{e}^{\mathrm{i} k r_{11}} \cdots \frac{1}{r_{n m}} \mathrm{e}^{\mathrm{i} k r_{n m}} \cdots \frac{1}{r_{N M}} \mathrm{e}^{\mathrm{i} k r_{N M}}\right] .
\end{aligned}
$$

When the sound from $\mathrm{L}$ point sources is incident on the cylindrical array, the data acquired by each element is the superposition of $\mathrm{L}$ point sound sources. The cross spectrum between $(1,1)$ and each of $N \times M$ elements are performed to get the complex sound pressure of the cylindrical array:

$$
\begin{gathered}
\boldsymbol{p}(r, \phi, z)= \\
{\left[p_{11}\left(r_{11}, \phi_{11}, z_{11}\right), \cdots, p_{n m}\left(r_{n m}, \phi_{n m}, z_{n m}\right),\right.} \\
\left.\cdots, p_{N M}\left(r_{N M}, \phi_{N M}, z_{N M}\right)\right]= \\
P_{0}\left[\frac{1}{r_{11}} \mathrm{e}^{\mathrm{i} k r_{11}} \cdots \frac{1}{r_{n m}} \mathrm{e}^{\mathrm{i} k r_{n m}} \cdots \frac{1}{r_{N M}} \mathrm{e}^{\mathrm{i} k r_{N M}}\right] .
\end{gathered}
$$

Double cylindrical array is composed of two cylindrical arrays with the same axis, and the radii of the two cylindrical array are $r_{H 1}$ and $r_{H 2}\left(r_{H 2}>r_{H 1}\right)$ respectively, the corresponding complex sound pressures are $p^{I}(r, \theta, \phi)$ and $p^{I I}(r, \theta, \phi)$ respectively.

The problem is to identify the noise source using the complex sound pressure data acquired by the double cylindrical array. The sound field separation technique is performed to separate the direct and reflected sounds. Noise source is located using near field acoustic holography in low frequency and using focused beamforming in high frequency.

\subsection{Sound Field Separation Technique Based on Double Cylindrical Array}

The outgoing wave and incoming wave are separated by using the sound field separation technique. Sound source of interest lying in the interior of the double cylindrical array generates outgoing wave, and the incoming wave coming from the exterior of double cylindrical array is generated by the reflection of the wall and ceiling. For testing the validity of the sound field separation technique, the sound source of interest lies in the interior of the double cylindrical array to be identified, and the external sound sources are looked as the interference sound sources of reflection, as shown in Fig. 3.

The radii of holography surfaces $S_{H 1}$ and $S_{H 2}$ are $r_{H 1}$ and $r_{H 2}$ respectively, and the distance between two holography surfaces is $d=r_{H 2}-r_{H 1}$. Sound source 1 of interest lies in the interior of holography surface $S_{H 1}$ and the sound source 2 of interference is out of the holography surface $S_{H 2}$. Sound pressures of holography surfaces $S_{H 1}$ and $S_{H 2}$ are as following: 


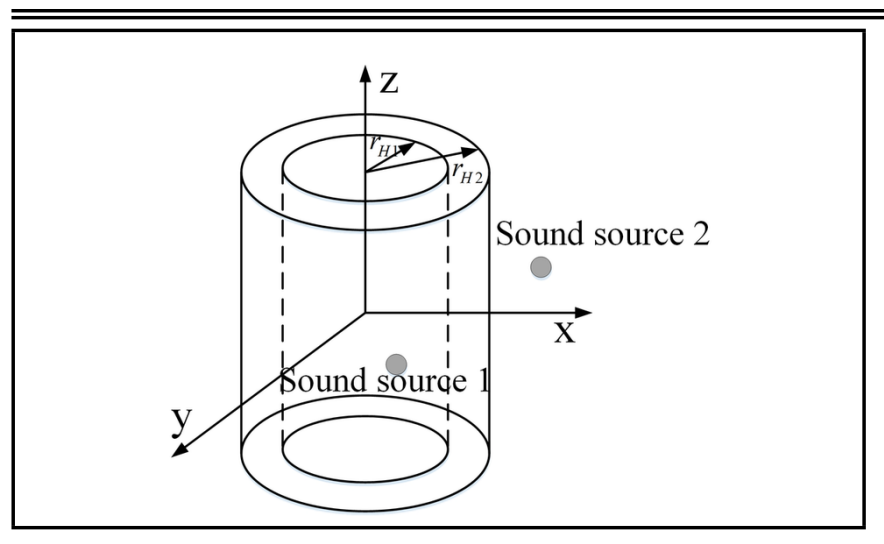

Figure 3. Sketch map of the sound field separation using double cylindrical array.

$$
\begin{aligned}
& p\left(r_{H 1}, \phi, z\right)=p^{I}\left(r_{H 1}, \phi, z\right)+p^{I I}\left(r_{H 1}, \phi, z\right) ; \\
& p\left(r_{H 2}, \phi, z\right)=p^{I}\left(r_{H 2}, \phi, z\right)+p^{I I}\left(r_{H 2}, \phi, z\right) ;
\end{aligned}
$$

where $p^{I}\left(r_{H 1}, \phi, z\right)$ and $p^{I}\left(r_{H 2}, \phi, z\right)$ represent, respectively, the sound pressures of sound source 1 incident on holography surfaces $S_{H 1}$ and $S_{H 2}$, as well as $p^{I I}\left(r_{H 1}, \phi, z\right)$ and $p^{I I}\left(r_{H 2}, \phi, z\right)$ represent, respectively, the sound pressure of sound source 2 incident on holography surfaces $S_{H 1}$ and $S_{H 2}$.

Fourier transform is performed on Eqs. (5) and (6). The relation of the sound pressure wave spectrum of two cylindrical holography surfaces is:

$$
\begin{aligned}
& P_{n}\left(r_{H 1}, k_{z}\right)=P_{n}^{I}\left(r_{H 1}, k_{z}\right)+P_{n}^{I I}\left(r_{H 1}, k_{z}\right) ; \\
& P_{n}\left(r_{H 2}, k_{z}\right)=P_{n}^{I}\left(r_{H 2}, k_{z}\right)+P_{n}^{I I}\left(r_{H 2}, k_{z}\right) .
\end{aligned}
$$

The relation of the sound pressure of two holography surfaces in wave number domain is:

$$
\begin{array}{r}
P_{n}{ }^{I}\left(r_{H 2}, k_{z}\right)=\frac{H_{n}^{(1)}\left(k_{r} r_{H 2}\right)}{H_{n}^{(1)}\left(k_{r} r_{H 1}\right)} P_{n}^{I}\left(r_{H 1}, k_{z}\right) ; \\
P_{n}{ }^{I I}\left(r_{H 1}, k_{z}\right)=\frac{H_{n}^{(2)}\left(k_{r} r_{H 1}\right)}{H_{n}^{(2)}\left(k_{r} r_{H 2}\right)} P_{n}^{I I}\left(r_{H 2}, k_{z}\right) .
\end{array}
$$

Combine Eqs. (7)- (10) to obtain Eqs. (11) and (12).

$P_{n}^{I}\left(r_{H 1}, k_{z}\right)$ is wave number domain sound pressure of holography surface I generated by sound source 1, and $P_{n}^{I I}\left(r_{H 2}, k_{z}\right)$ is the wave number domain sound pressure of holography surface II generated by sound source 2 . Outgoing wave and incoming wave are separated, and cylindrical near field acoustic holography and focused beamforming are performed to locate sound sources of interest.

\subsection{Cylindrical Near Field Acoustic Holography}

The cylindrical wave spectrum of reconstructed cylindrical surface with $r=r_{S}$ is obtained as:

$$
P_{n}\left(r_{S}, k_{z}\right)=\frac{H_{n}^{(1)}\left(k_{r} r_{S}\right)}{H_{n}^{(1)}\left(k_{r} r_{H}\right)} P_{n}^{I}\left(r_{H 1}, k_{z}\right) .
$$

The sound pressure of reconstructed cylindrical surface $p\left(r_{s}, \phi, z\right)$ with $r=r_{s}$ is obtained by space inverse Fourier transform of $P_{n}\left(r_{S}, k_{z}\right)$ as:

$$
p\left(r_{S}, \phi, z\right)=F_{\phi}^{-1} F_{z}^{-1}\left[P_{n}\left(r_{S}, k_{z}\right)\right] .
$$

The noise source identification for the interior source of cylindrical surface when $r_{H}>r_{S}$, corresponds to the reconstructed process with sensitiveness to noise in high wave number. The measurement error will be extremely amplified in high wave number $k_{z}$ and $n / r$. The filter function is used to restrain the error amplification of high frequency wave number as:

$$
W\left(n, k_{z}\right)=\left\{\begin{array}{ll}
1-\frac{1}{2} e^{\left(k_{r} / k_{c}-1\right) / \alpha} & k_{r} \leq k_{c} \\
\frac{1}{2} e^{\left(1-k_{r} / k_{c}\right) / \alpha} & k_{r}>k_{c}
\end{array} ;\right.
$$

where $k_{c}$ is the cut-off wave number of filter $k_{r}=$ $\sqrt{k_{z}^{2}+\left(n / r_{H}\right)^{2}} ; \alpha$ is the steep coefficient window function. The smaller the value of $\alpha$, the steeper the cutoff of the function value at $k_{c}$. The sound pressure of reconstructed cylindrical surface is obtained by two-dimensional Fourier transforms of the filtered cylindrical wave spectrum. The total expression of cylindrical surface near field acoustic holography is given by:

$$
\begin{aligned}
& p\left(r_{S}, \phi, z\right)= \\
& \quad F_{\phi}^{-1} F_{z}^{-1}\left\{W\left(n, k_{z}\right) \frac{H_{n}^{(1)}\left(k_{r} r_{S}\right)}{H_{n}^{(1)}\left(k_{r} r_{H}\right)} P_{n}^{I}\left(r_{H 1}, k_{z}\right)\right\} .
\end{aligned}
$$

The reconstruction surface is chosen to contain the sound source for sound source identification.

\subsection{Focused Beamforming of Cylindrical Array}

Focused beamforming is performed to identify sound source in high frequency. Conventional beamforming uses pressure data measured by single measuring surface to locate the sound source. When target sound sources and interference sound sources appear at different sides of the measuring surface at the same time, the interference sound source generates the false source on the reconstruction surface, so that the target sound source cannot be accurately identified. The double layer beamforming technique can be used to solve this problem. ${ }^{23,24}$ The particle velocity is obtained by the Euler formula and the finite difference theory, and sound intensity is obtained by multiplying the particle velocity and the sound pressure.

The section of the double cylindrical array is shown in Fig. 4. $R_{C n m}$ is the distance from the sound source to the middle point of the number $(n, m)$ microphones on the two holography surfaces. $S_{C}$ is the surface of the middle points of the number $(n, m)$ microphones on the two holography surfaces, $\theta_{C n m}$ is the angle between $R_{C n m}$ and the horizontal plane. $d$ is the distance between two holography surfaces.

By using the Euler formula and the finite difference theory, particle velocity approximation can be obtained as: 


$$
\begin{aligned}
& P_{n}^{I}\left(r_{H 1}, k_{z}\right)=\frac{P_{n}\left(r_{H 1}, k_{z}\right) H_{n}^{(1)}\left(k_{r} r_{H 1}\right) H_{n}^{(2)}\left(k_{r} r_{H 2}\right)-P_{n}\left(r_{H 2}, k_{z}\right) H_{n}^{(2)}\left(k_{r} r_{H 1}\right) H_{n}^{(1)}\left(k_{r} r_{H 1}\right)}{H_{n}^{(1)}\left(k_{r} r_{H 1}\right) H_{n}^{(2)}\left(k_{r} r_{H 2}\right)-H_{n}^{(1)}\left(k_{r} r_{H 2}\right) H_{n}^{(2)}\left(k_{r} r_{H 1}\right)} \\
& P_{n}^{I I}\left(r_{H 2}, k_{z}\right)=\frac{P_{n}\left(r_{H 2}, k_{z}\right) H_{n}^{(1)}\left(k_{r} r_{H 1}\right) H_{n}^{(2)}\left(k_{r} r_{H 2}\right)-P_{n}\left(r_{H 1}, k_{z}\right) H_{n}^{(1)}\left(k_{r} r_{H 2}\right) H_{n}^{(2)}\left(k_{r} r_{H 2}\right)}{H_{n}^{(1)}\left(k_{r} r_{H 1}\right) H_{n}^{(2)}\left(k_{r} r_{H 2}\right)-H_{n}^{(1)}\left(k_{r} r_{H 2}\right) H_{n}^{(2)}\left(k_{r} r_{H 1}\right)} .
\end{aligned}
$$

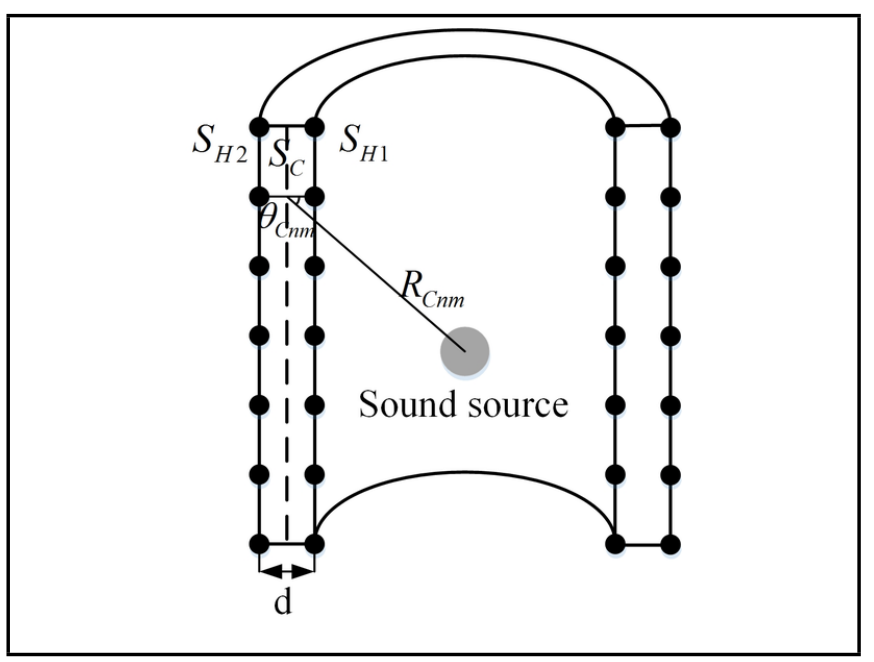

Figure 4. Sketch map of the double cylindrical array.

$$
\begin{aligned}
& u\left(r_{C n m}, \phi_{C n m}, z_{C n m}\right) \approx \\
& \frac{p\left(r_{H 2 n m}, \phi_{H 2 n m}, z_{H 2 n m}\right)-p\left(r_{H 1 n m}, \phi_{H 1 n m}, z_{H 1 n m}\right)}{-\mathrm{j} k \Delta R_{C n m} \rho_{0} c} ;
\end{aligned}
$$

where $\Delta R_{C n m}=d \cos \theta_{C m n}$.

$$
\begin{aligned}
& \boldsymbol{u}(r, \phi, z)= \\
& {\left[u\left(r_{C 11}, \phi_{C 11}, z_{C 11}\right), \cdots, u\left(r_{C n m}, \phi_{C n m}, z_{C n m}\right),\right.} \\
& \left.\cdots, u\left(r_{C N M}, \phi_{C N M}, z_{C N M}\right)\right] .
\end{aligned}
$$

The steering vector $\boldsymbol{E}$ of focused beamforming is:

$$
\boldsymbol{E}=\left[r_{11} \mathrm{e}^{\mathrm{i} k r_{11}}, \cdots, r_{n m} \mathrm{e}^{\mathrm{i} k r_{n m}}, \cdots, r_{N M} \mathrm{e}^{\mathrm{i} k r_{N M}}\right]
$$

The output of focused beamforming using pressure and particle velocity of cylindrical array are:

$$
\begin{aligned}
\boldsymbol{B}_{p} & =\frac{1}{M \cdot N}\left|\left\langle\boldsymbol{p}(r, \phi, z) \cdot \boldsymbol{E}_{p}\right\rangle\right| ; \\
\boldsymbol{B}_{u} & =\frac{1}{M \cdot N}\left|\left\langle\boldsymbol{u}(r, \phi, z) \cdot \boldsymbol{E}_{u}\right\rangle\right| ;
\end{aligned}
$$

where $\langle\cdot\rangle$ represents the inner product of two vectors. The steering vectors of sound pressure and particle velocity are $\boldsymbol{E}_{p}$ and $\boldsymbol{E}_{u}$, respectively, that are similar to Eq. (19) corresponding to the distance from scan surface to surface $S_{H 1}$ and $S_{C}$ as shown in Fig. 4. Next, $1 /(M \cdot N)$ is factor for uniformation of element number. Focused beamforming scans all the discrete points in interesting region, and the amplitude and phase of signals of different elements are compensated simultaneously.
If the scan region includes the sound source points, when the scan point coincides with the sound source point, the sound source will be located. Sound intensity beamforming can be obtained as following:

$$
\boldsymbol{S}_{p u}=\frac{1}{2} \operatorname{Re}\left(\boldsymbol{B}_{p} \boldsymbol{B}_{u}^{*}\right) .
$$

To quantify the location results, the positioning error is defined, expressed as:

$$
l=z_{c}-z_{t}
$$

where $z_{t}$ is the theoretical location of the sound source, $z_{c}$ is the position located by an algorithm. In NAH, the Fourier transform of the discrete space can get the sound pressure value at the discrete points on the reconstructed surface. For the two methods to be combined, the scanning point in focused beamforming should be the same as the position of the discrete points on the reconstructed surface. Therefore, the positioning error on the cylinder is affected by the position of the discrete points, and the positioning error smaller than the position of the discrete points can't be obtained accurately. The interval between discrete points in this simulation is $0.05 \mathrm{~m}$. Positioning error is an integer multiple of $0.05 \mathrm{~m}$.

\subsection{Frequency Band Separation for Near Field Acoustic Holography and Focused Beamforming}

The suitable frequency bands of cylindrical near field acoustic holography and focused beamforming are studied. The space distinguish ability for near field acoustic holography is about a constant with relation of measurement distance and signal to noise ratio in low frequency, and generally equal to half of the wave length of signal in high frequency. The space distinguish ability of focused beamforming is larger than a wave length in low frequency. In low frequency band, the space distinguish ability of near field acoustic holography is superior to that of focused beamforming. In high frequency band, near field acoustic holography needs more microphones and shorter distance of microphones to get high space distinguish ability, which is difficult to achieve. Therefore, the sound source is located using near field acoustic holography in low frequency region and using focused beamforming in high frequency region.

The upper limit frequency of near field acoustic holography acts as the divided frequency point of near field acoustic holography and focused beamforming. If the distance of element is $\Delta$, according to Nyquist space sample theorem, the maximum wave number measured is $k_{\max }=\pi / \Delta$, with the interference of noise in practice $k_{\max }<\pi / \Delta$; thus, the maximum frequency of reconstructed signals using near field acoustic holography is:

$$
f_{\max }<\frac{\pi c}{2 \pi \Delta}=\frac{c}{2 \Delta} .
$$


The distance of elements in circumference direction is $\Delta \phi$ and the distance of elements in axis direction is $\Delta z$, we get:

$$
\begin{gathered}
f_{\Delta z \max }<\frac{c}{2 \Delta z} ; \\
f_{\Delta \phi \max }<\frac{c}{\left.2 \Delta \phi \cdot r_{H}\right)} .
\end{gathered}
$$

The identification performance of near field acoustic holography is also related to the measurement distance. The evanescent wave attenuates in accordance with exponent rule. The measurement distance is less than $1 / 3$ wavelength to effectively measure the evanescent wave, so the upper frequency limit has relation to the measurement distance as following:

$$
f_{d \max }<\frac{c}{3 d}
$$
is

The upper frequency limit of near field acoustic holography

$$
f_{\max }=\left|f_{\Delta z \max }, f_{\Delta \phi \max }, f_{d \max }\right|_{\min } .
$$

The frequency range of near field acoustic holography and focused beamforming is divided according to Eq. (28).

\section{SIMULATIONS}

The performance of sound source identification using double cylindrical array is simulated. The elements are uniformly delivered along circumference direction and axis direction. There are 31 elements along axis direction with inner distance $\Delta z=0.05 \mathrm{~m}$ and 36 elements along circumference direction with angle distance $\Delta \phi=10$. The number of each cylindrical array is $31 \times 36=1116$ and the radii of double cylindrical array are $r_{H 1}=0.25 \mathrm{~m}$ and $r_{H 2}=0.3 \mathrm{~m}$ respectively. The distance between the two holography surfaces is $0.05 \mathrm{~m}$.

The upper limit frequency is determined by $\Delta z=0.05 \mathrm{~m}$, $\Delta \phi=10^{\circ}$ and measurement distance $d$, we obtain:

$$
\begin{gathered}
f_{\Delta z \max }<\frac{c}{2 \Delta z}=\frac{340}{2 \cdot 0.05}=3400 \mathrm{~Hz} \\
f_{\Delta \phi \max }<\frac{c}{\left.2 \Delta \phi \cdot \frac{\pi}{180} \cdot r_{H}\right)}=\frac{340}{2\left(10 \cdot \frac{\pi}{180} \cdot 0.3\right)} \approx 3200 \mathrm{~Hz} \\
f_{d \max }<\frac{c}{3 \cdot d}=\frac{340}{3 \cdot 0.075} \approx 1500 \mathrm{~Hz}
\end{gathered}
$$

The upper frequency limit of near field acoustic holography is the minimum of $f_{\Delta z \max }, f \Delta_{\phi \max }$ and $f_{d \max }$. We get $f_{\max }=1500 \mathrm{~Hz}$. When the frequency is lower than $1500 \mathrm{~Hz}$, near field acoustic holography is used to identify the sound source. When the frequency is higher than $1500 \mathrm{~Hz}$, focused beamforming will be used to locate the sound source.

\subsection{Simulations of Location of a Single Sound Source and Vibrating Cylindrical Structure}

The performance of sound source identification for a single source is simulated. The sound source of interest lies in the interior of the cylindrical array and the cylindrical coordinate of sound source is $\left(0.2 \mathrm{~m}, 0^{\circ}, 0 \mathrm{~m}\right)$. There are two interference

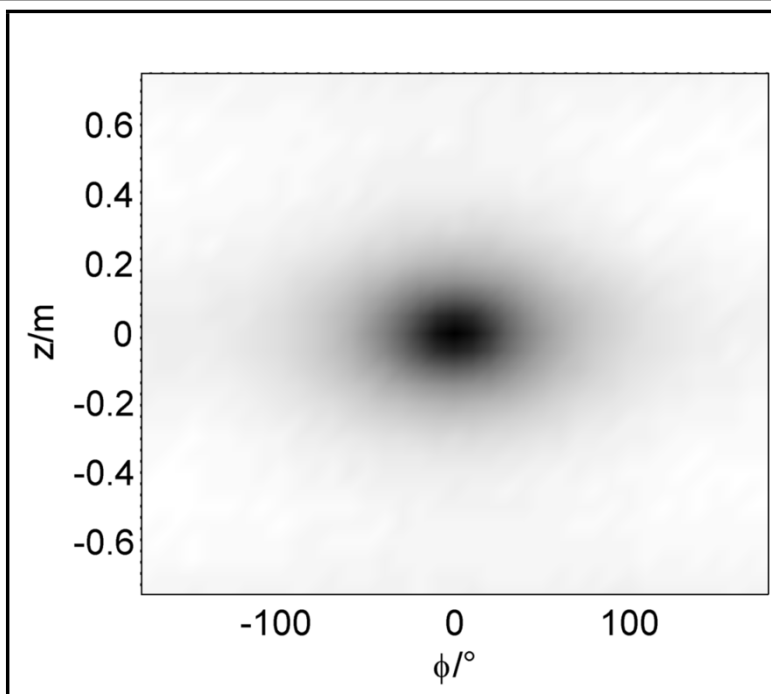

(a) Sound pressure distribution after separation

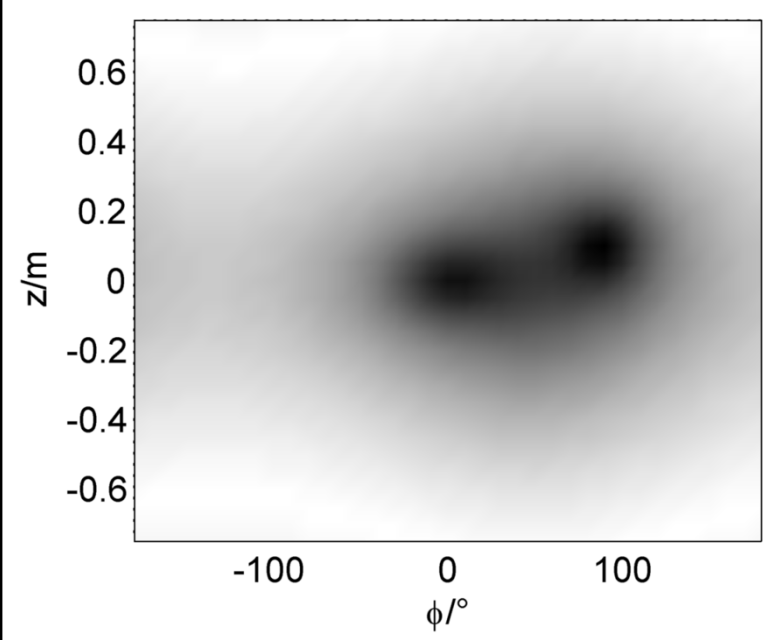

(b) Sound pressure distribution of measurement.

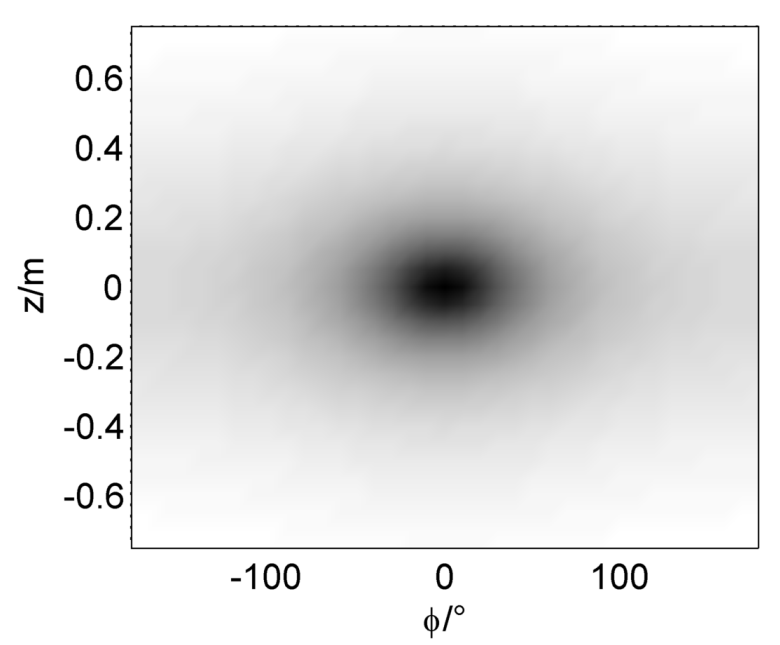

(c) Sound pressure distribution of theory calculation.

Figure 5. Comparison of sound pressure distribution of cylindrical holography $S_{H 1}$.

sources in the exterior of the cylindrical array with cylindrical coordinates of $\left(0.56 \mathrm{~m}, 45^{\circ},-0.1 \mathrm{~m}\right)$ and $\left(0.35 \mathrm{~m}, 90^{\circ}, 0.1 \mathrm{~m}\right)$, and frequency of signal of interest and interference sources is $f=400 \mathrm{~Hz}$, with Gaussian noise and SNR $=40 \mathrm{~dB}$.

To quantify the separation result, the relative root-mean- 
square errors of the theoretical sound pressure value on holography surface and sound pressure value after separation on the same surface are defined as the separation error, expressed as:

$$
e r r=\sqrt{\frac{\sum\left|p_{t h}-\hat{p}\right|^{2}}{\sum\left|p_{t h}\right|^{2}}} \times 100 \%
$$

where $p_{t h}$ is the theoretical sound pressure value and $\hat{p}$ is the sound pressure value after separation. The sound field separation is performed to remove the effect of outer interference sources. For testing the validity of sound field separation technique, the sound pressure distribution of separation and measurement are demonstrated in Figs. 5a and 5b. For comparison, the sound pressure distribution of theory calculation without the interference sources is shown in Fig. 5c. The results indicate that the sound field separation technique can effectively remove the effect of exterior interference sources. The separation error is $33 \%$. The sound pressure data after separation will be used for further noise source identification.

The performance of a single source identification using near field acoustic holography and focused beamforming in different frequency is researched. The frequency of signals successively changes to $1000 \mathrm{~Hz}, 1500 \mathrm{~Hz}$, and $3000 \mathrm{~Hz}$; the other simulation conditions remain the same as before. The sound source identification results of near field acoustic holography and focused beamforming are demonstrated in Fig. 6. The left portion of each row is a grey-scale map of near field acoustic holography identification result with the pressure magnitude indicated by the shade; increase in darkness indicates increase in pressure magnitude. The right one is the identification result of focused beamforming. For comparison, the theory position of the sound source of interest is shown by the "+" in each map.

Figure 6 shows that $f=1500 \mathrm{~Hz}$ is taken as the frequency separation point. Sound source can be effectively identified using near field acoustic holography in low frequency and using focused beamforming in high frequency. High resolution sound source localization can be obtained. The positioning error of single source localization is within $0.05 \mathrm{~m}$.

The performance of a single source with wide frequency band identification is researched. The frequency of signals is $100 \mathrm{~Hz}-4000 \mathrm{~Hz}$; the other simulation conditions remain the same as before. The sound source is located using the combined method; when the frequency is below $1500 \mathrm{~Hz}, \mathrm{NAH}$ is used, and when it is above $1500 \mathrm{~Hz}$, beamforming is used. The sound source identification result is demonstrated in Fig. 7. The result shows that a sound source with a wide frequency band can be effectively identified and distinguished by using the combined method.

Vibrating cylindrical structure is typical application for $\mathrm{NAH}$ in cylindrical coordinates. So, the performance of NAH for a Vibrating cylindrical structure is simulated. A cylindrical shell with a radius of $0.8 \mathrm{~m}$ and a length of $2 \mathrm{~m}$ is simulated in COMSOL. A cylinder with a radius of $0.2 \mathrm{~m}$ and a length of $0.5 \mathrm{~m}$ was placed in the cylindrical shell. Figure 8 shows that there are some point sources on the cylinder to stimulate it, which form a vibrating cylindrical structure. The sound pressure distribution of theory calculation on the surface of cylinder is shown in Fig. 9. The sound source identification result of near field acoustic holography is demonstrated in Fig. 10. The results show that the vibrating cylindrical structure can be effectively identified and distinguished by using near field acoustic holography.

\subsection{Distinction between Two Sound Sources}

There are two sound sources of interest that lie in the interior of the cylindrical array with the cylindrical coordinates of $\left(0.15 \mathrm{~m}, 0^{\circ}, 0 \mathrm{~m}\right)$ and $\left(0.15 \mathrm{~m}, 90^{\circ}, 0 \mathrm{~m}\right)$. The frequency of signals is both $100 \mathrm{~Hz}$, keeping other simulation conditions the same as the last simulation in Fig. 6. Then the frequency of signals change to $4000 \mathrm{~Hz}$, keeping other simulation conditions the same as before. The results show that multi-sound sources can be effectively identified and distinguished by using near field acoustic holography in low frequency and focused beamforming in high frequency.

The sound sources are changed to prove the effectiveness of the combined method. The frequency of sound source with the coordinates of $\left(0.15 \mathrm{~m}, 0^{\circ}, 0 \mathrm{~m}\right)$ changes to $100 \mathrm{~Hz}$. The frequency of sound source with the coordinates of $\left(0.15 \mathrm{~m}, 90^{\circ}\right.$, $0 \mathrm{~m}$ ) changes to $4000 \mathrm{~Hz}$ and the other simulation conditions remain the same as before. The sound source identification results using near field acoustic holography and focused beamforming are demonstrated, respectively, in Figs. 11 and 12. The sound source identification result using the combined method of near field acoustic holography and focused beamforming is demonstrated in Fig. 13. The output by using NAH in the low frequency band is added to the output by using beamforming in the high frequency band. The Fig. 13 is a summation of Fig. 11 and Fig. 12.

Comparison of Figs. 11, 12, and 13 shows that the sound sources fail to be distinguished by only using near field acoustic holography or only using focused beamforming when the sound sources include high frequency and low frequency signals. The combined method can effectively distinguish and locate the two sound sources.

\section{EXPERIMENT}

\subsection{Test Setup}

The objective here is to demonstrate the usefulness of the combined method of near field acoustic holography and focused beamforming in identification noise source in an enclosed space. The experiment of noise source identification using the double cylindrical array in submarine cabin model was carried out. Two loudspeakers as two sound sources were placed in the interior of double cylindrical array. The direct radiation sound went out from the interior of the double cylindrical array, and the reflection sound reflected by the walls and ceiling went in from the exterior of the double cylindrical array. The double cylindrical array comprises 62 microphones and a steel frame. Two-line arrays constructed with $62(31 \times 2)$ microphones were connected with the steel frame. The steel frame was fixed in the submarine cabin model and it could rotate around one side of the frame to form a double cylindrical array. The distribution of the double cylindrical scan array and sound sources in submarine cabin model is shown in Fig. 14. The diameters of the inner cylindrical array and outer cylindrical array were $0.75 \mathrm{~m}$ and $0.85 \mathrm{~m}$, respectively. The length of the cylindrical array was $1.5 \mathrm{~m}$. There are 31 elements of each cylindrical array along the axis direction with inner distance 


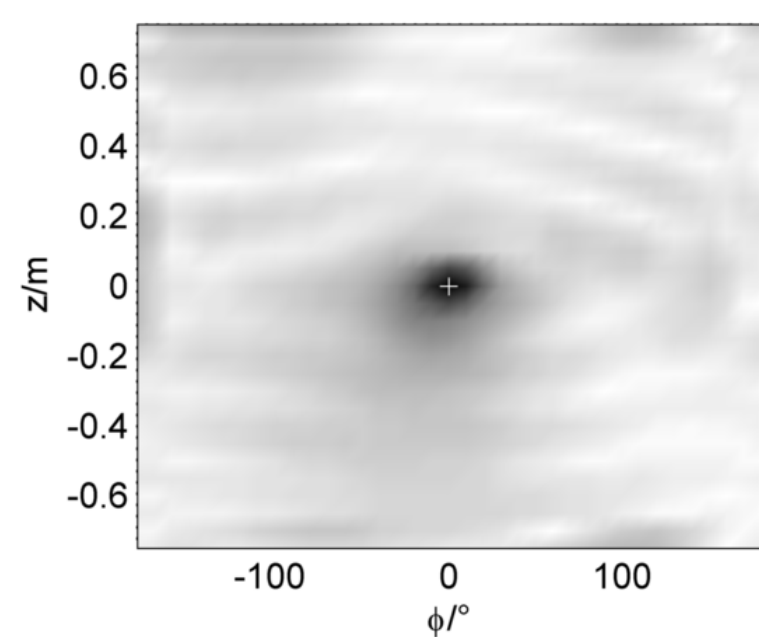

(a) Near field acoustic holography.

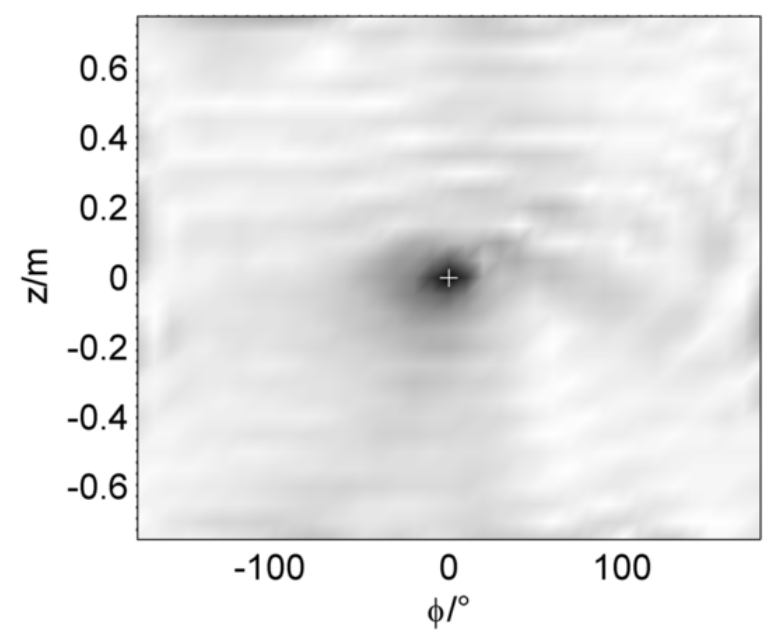

(c) Near field acoustic holography.

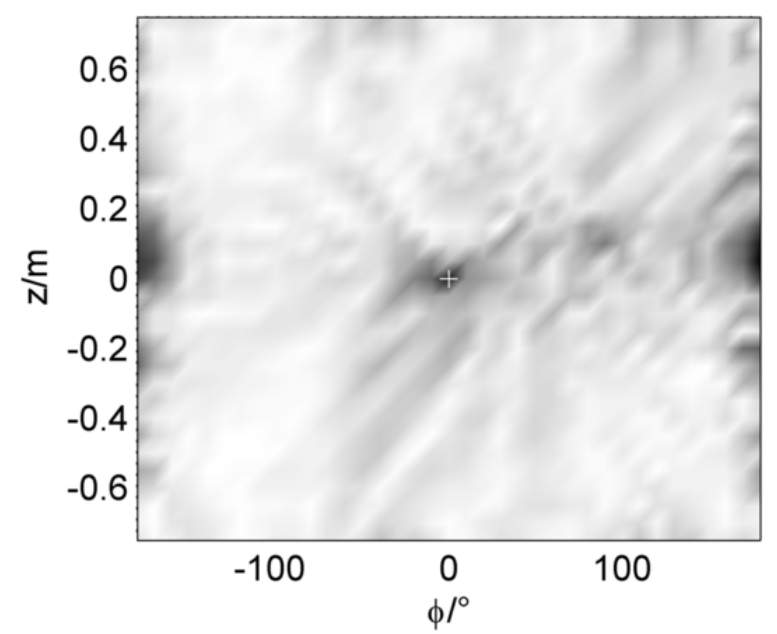

(e) Near field acoustic holography.

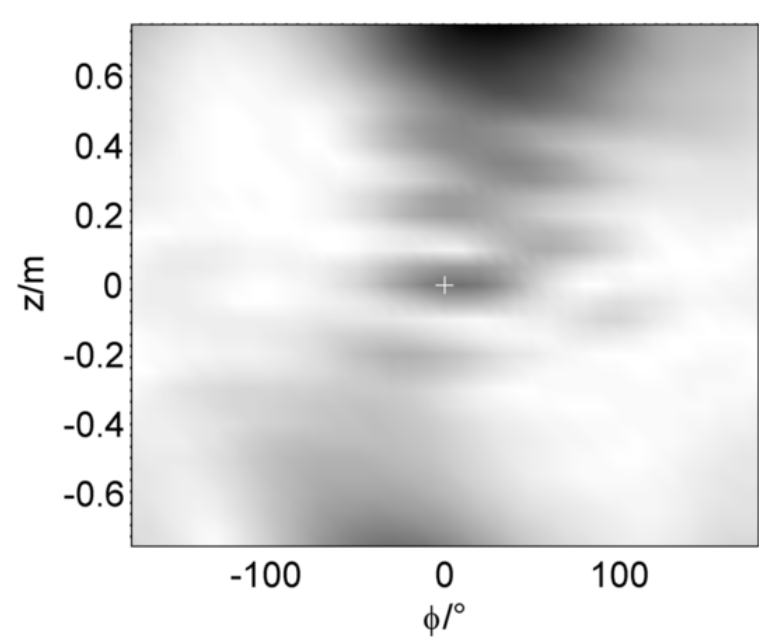

(b) Focused beamforming $(f=1000 \mathrm{~Hz})$.

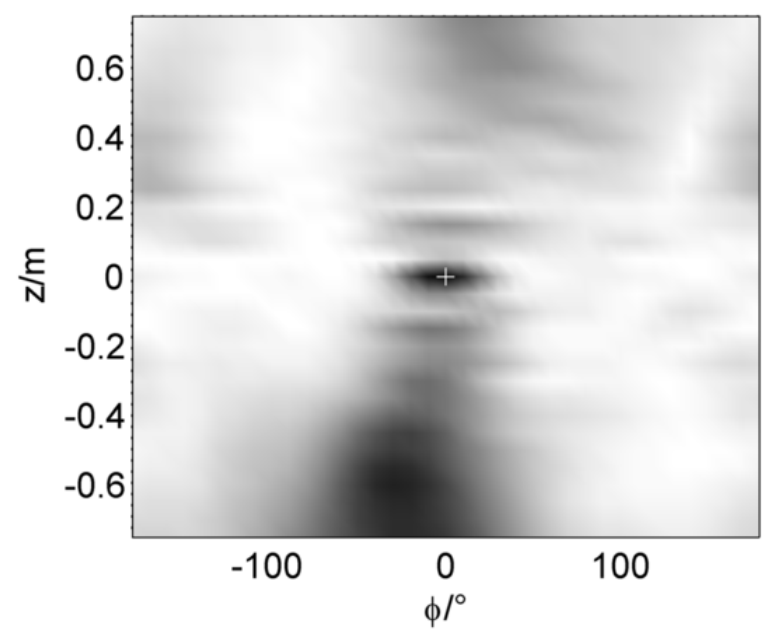

(d) Focused beamforming $(f=1500 \mathrm{~Hz})$.

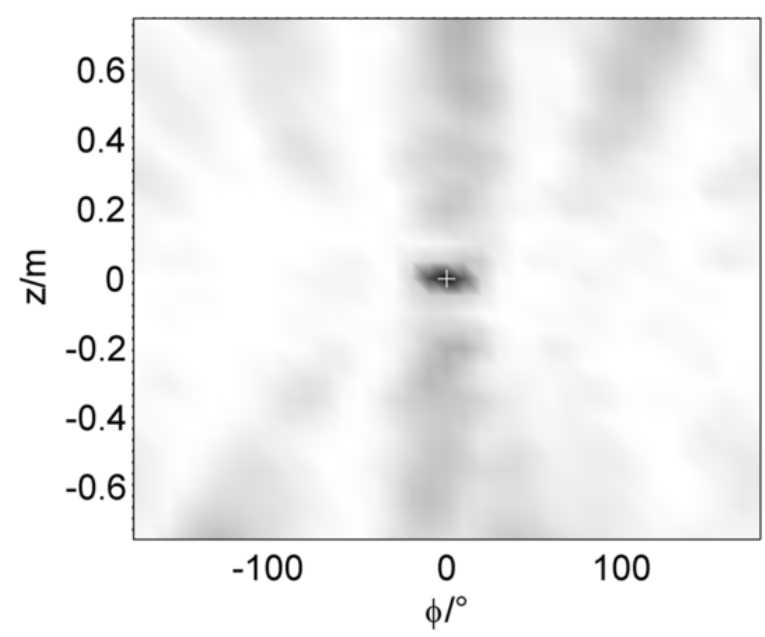

(f) Focused beamforming ( $f=3000 \mathrm{~Hz}$ ).

Figure 6. Comparison of sound pressure distribution of cylindrical holography $S_{H 1}$. 


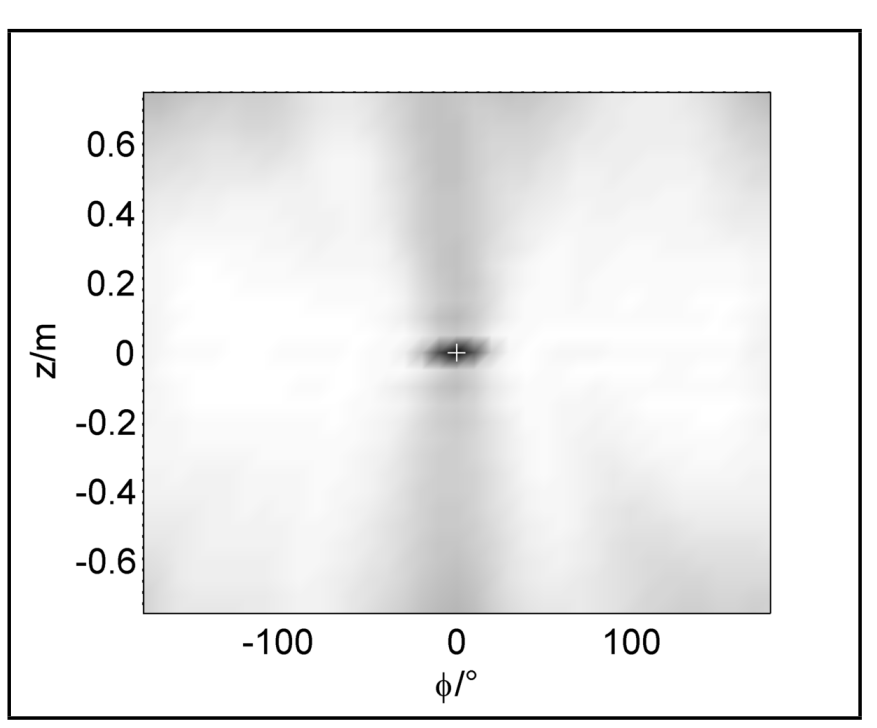

Figure 7. Sound source identification result of wide frequency band.

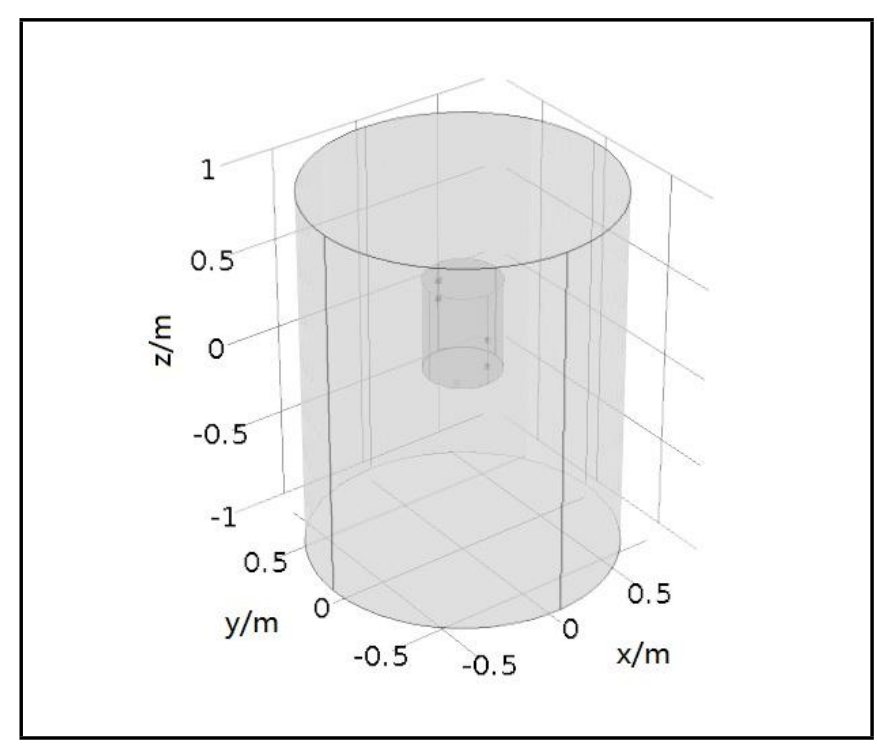

Figure 8. Vibrating cylindrical structure in the cylindrical shell.

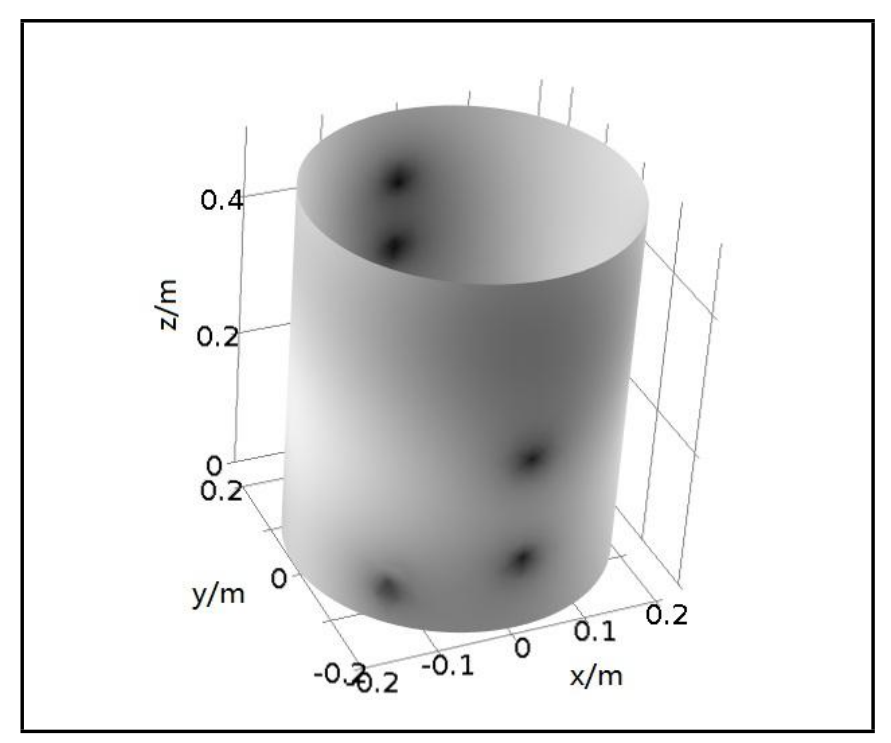

Figure 9. Sound pressure distribution of theory calculation in COMSOL.

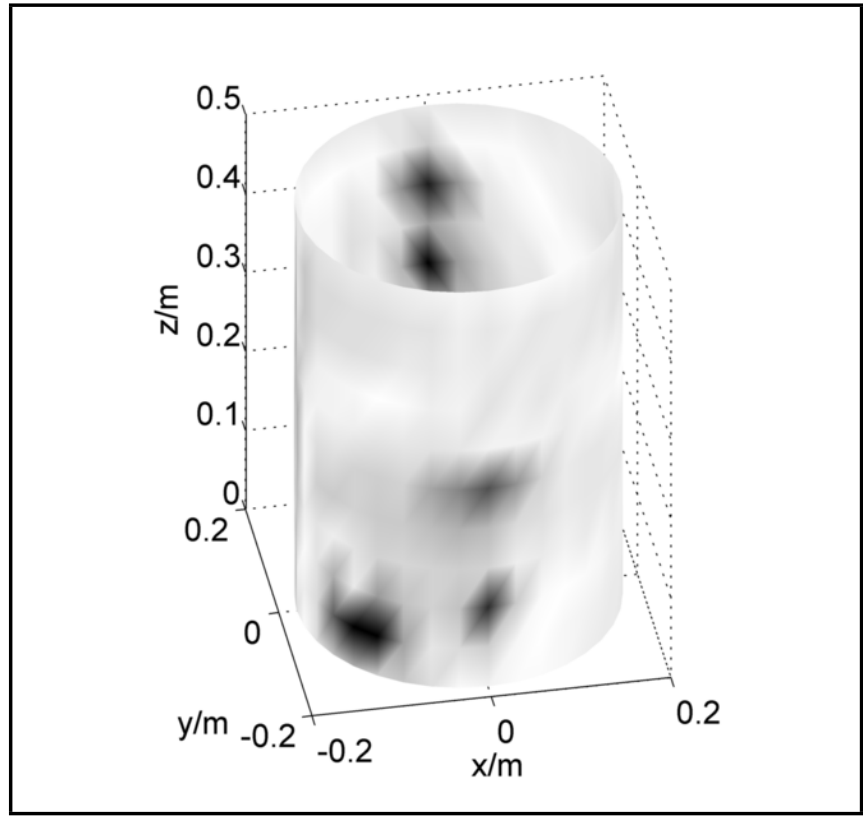

Figure 10. Sound source identification result of near field acoustic holography.

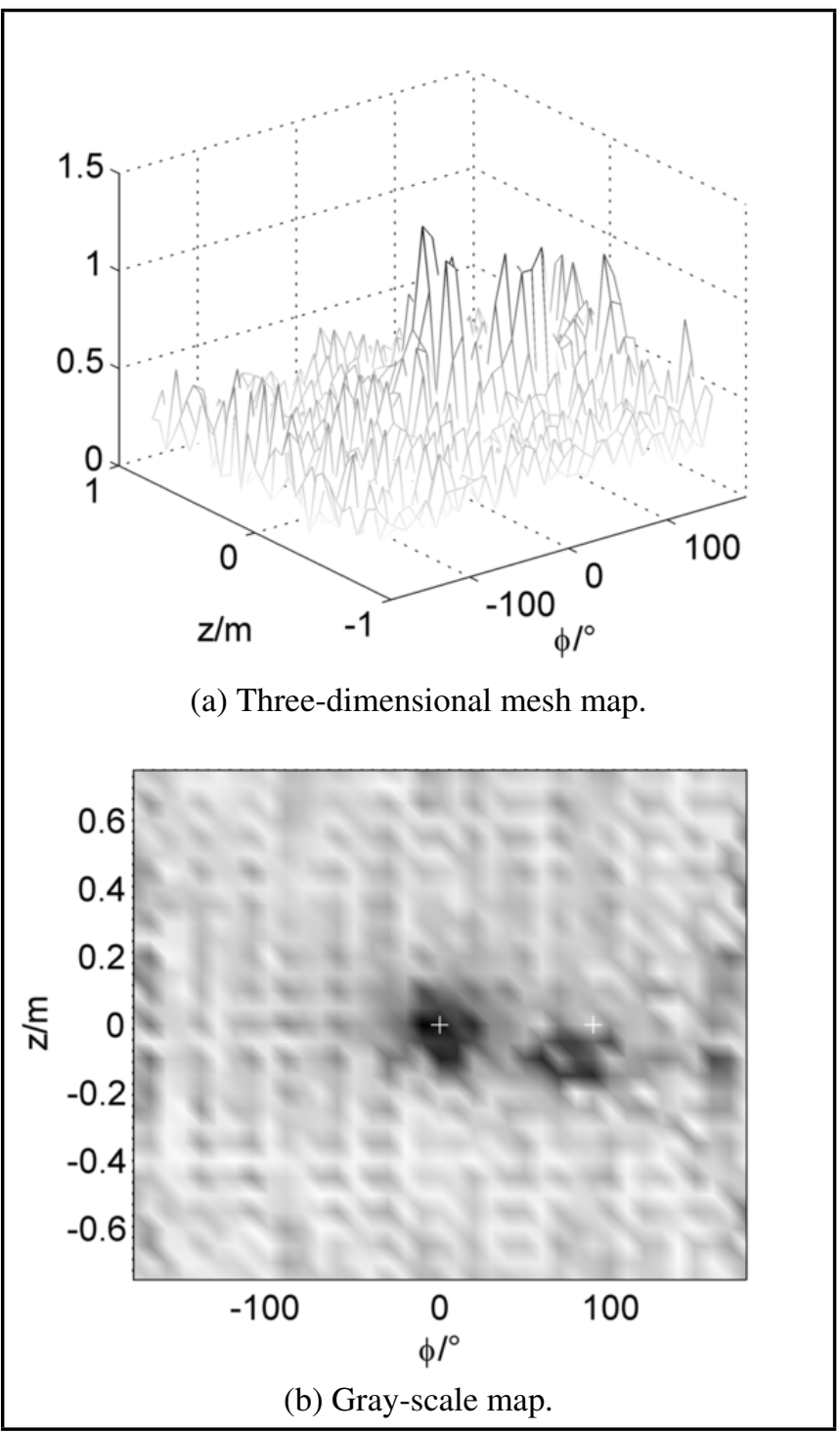

Figure 11. Sound source identification using near field acoustic holography. $\left(f_{1}=100 \mathrm{~Hz}, f_{2}=4000 \mathrm{~Hz}\right)$. 


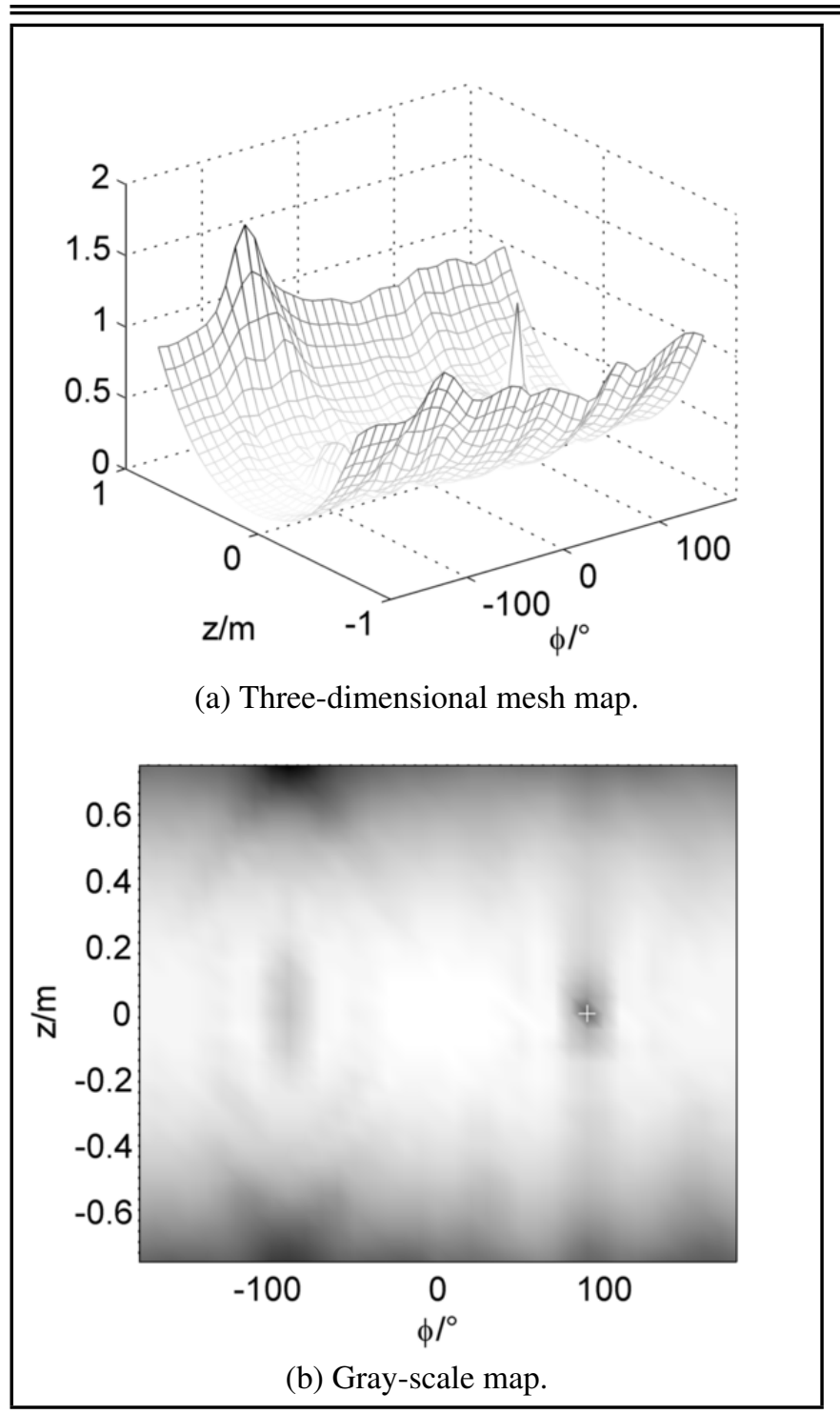

Figure 12. Sound source identification using focused beamforming. $\left(f_{1}=\right.$ $100 \mathrm{~Hz}, f_{2}=4000 \mathrm{~Hz}$ ).

$\Delta z=0.05 \mathrm{~m}$. The steel frame rotates clockwise with 10 interval to complete cylindrical scanning. A reference microphone is used to combine data from the different scan positions into a complete cylinder data. The position of the reference microphone is fixed. Then the complete cylinder data is used to locate the sound source by using NAH and focused beamforming.

\subsection{Experiment Results}

There were two sound sources of interest in the interior of the cylindrical array with the cylindrical coordinates of $(0.3 \mathrm{~m}$, $\left.10^{\circ},-0.15 \mathrm{~m}\right)$ and $\left(0.3 \mathrm{~m}, 10^{\circ}, 0.15 \mathrm{~m}\right)$. The frequency of signals was $2400 \mathrm{~Hz}$ and $315 \mathrm{~Hz}$, respectively. The sound source identification results using near field acoustic holography after sound field separation was demonstrated in Fig. 15 and that using focused beamforming was shown in Fig. 16. The sound source identification result using the combined method of near field acoustic holography and focused beamforming was demonstrated in Fig. 17.

Figure 15 shows that sound source can be located with low frequency and fails to be located with high frequency when using near field acoustic holography. Figure 16 demonstrates that sound source can be located with high frequency and fails to

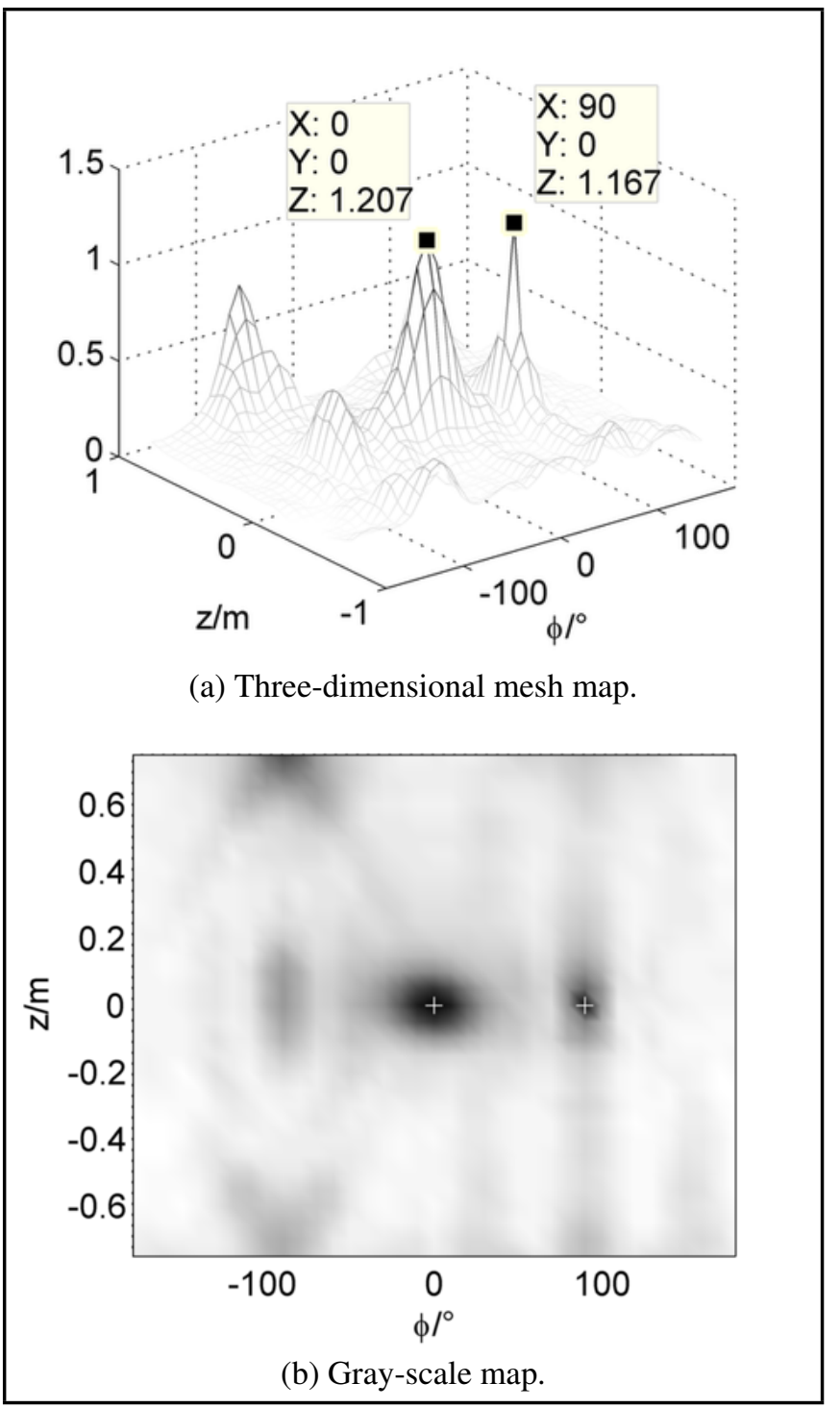

Figure 13. Sound source identification using the combined method. $\left(f_{1}=\right.$ $100 \mathrm{~Hz}, f_{2}=4000 \mathrm{~Hz}$ ).

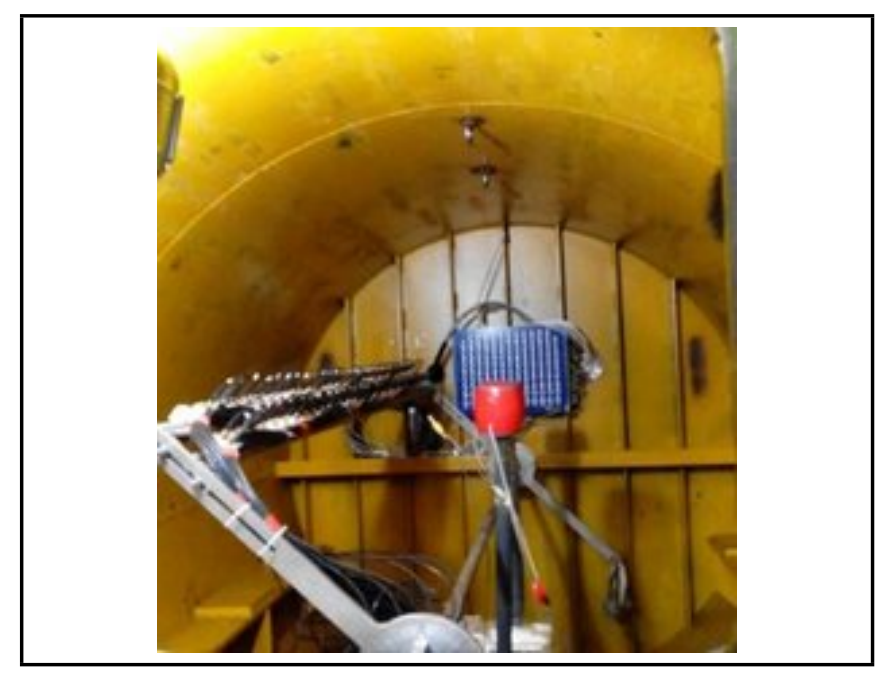

Figure 14. Double cylindrical scan array and sound sources in submarine cabin model. 


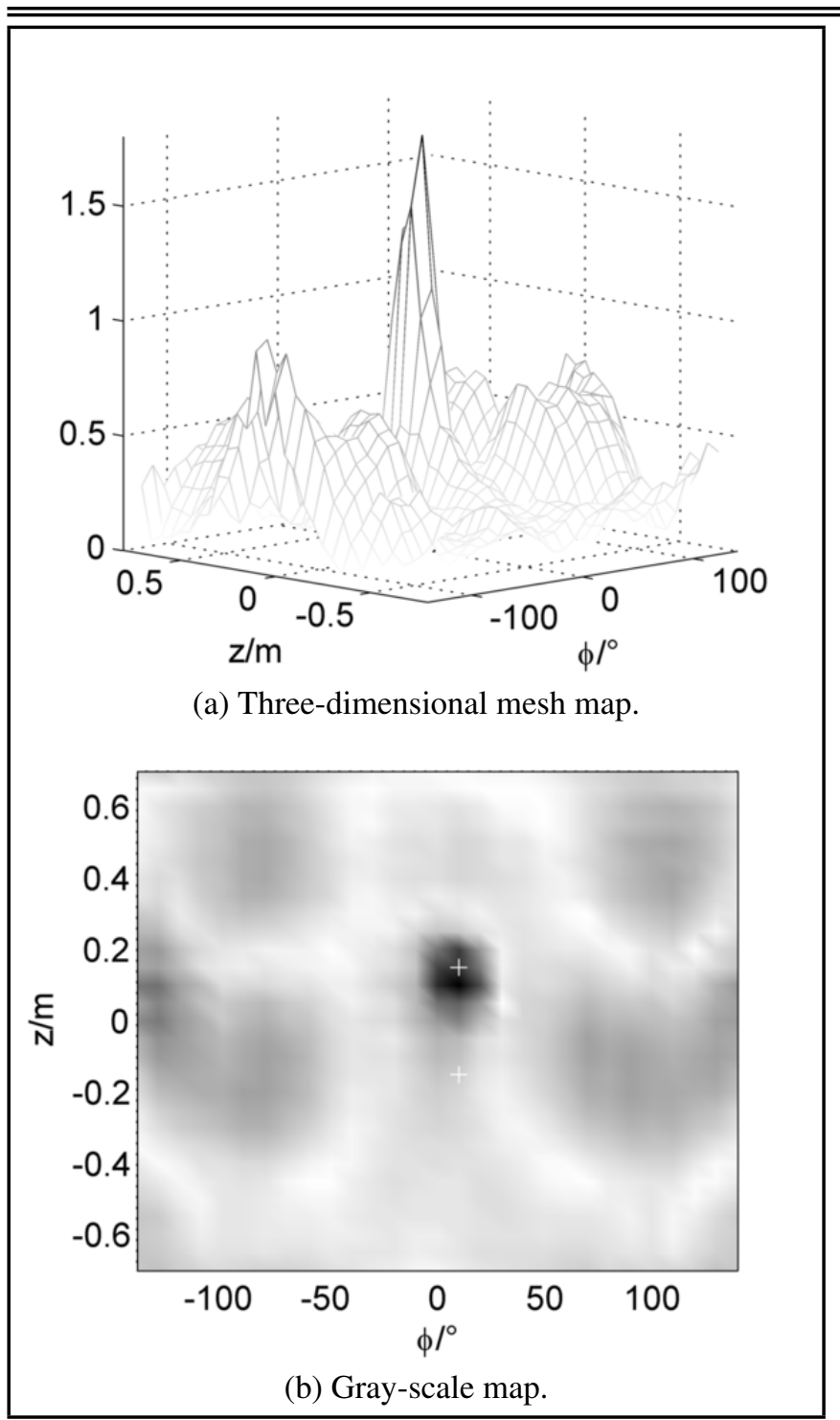

Figure 15. Sound source identification using near field acoustic holography.

be located with low frequency when using focused beamforming. Figure 17 indicates that the combined method of near field acoustic holography and focused beamforming can locate and distinguish sound sources with low and high frequencies in the submarine cabin model. The validity of sound field separation and combined method in practice were proved.

\section{CONCLUSIONS}

Sound source identification in enclosed space is researched in this paper. The combined method is studied for sound source identification of low and high frequencies. The divided frequency point can be calculated by Eqs. (25), (26), and (27). The upper limit frequency of the method is affected by the side lobes. The simulation and experimental results indicate that the effect of reflection sound can be removed using sound field separation technique, and high distinguish ability can be obtained for both high and low frequency signals by using combined method of near field acoustic holography and focused beamforming. The positioning error of source localization is $0.05 \mathrm{~m}$ with double cylindrical array in enclosed space. The studying results render the technical support for noise control in enclosed spaces.

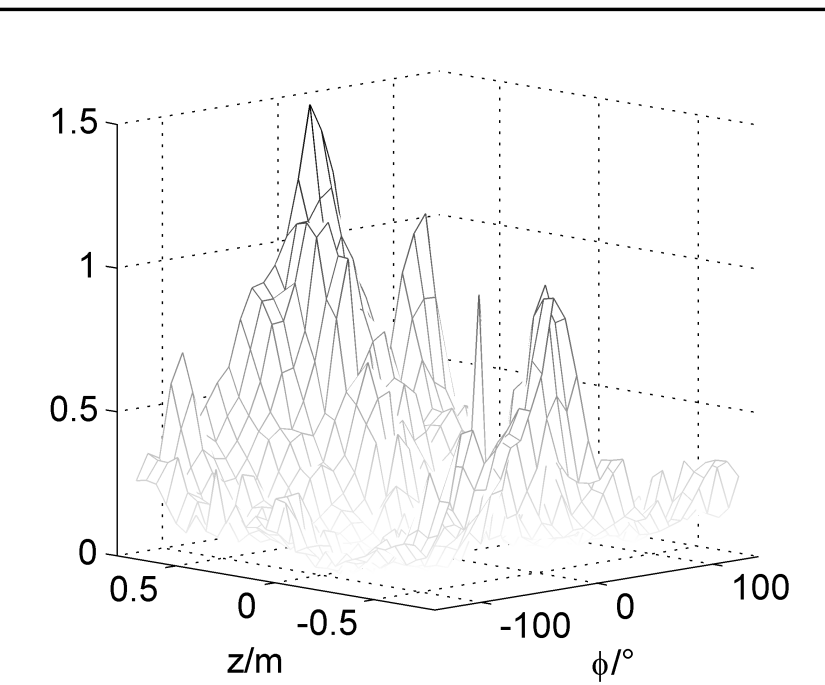

(a) Three-dimensional mesh map.

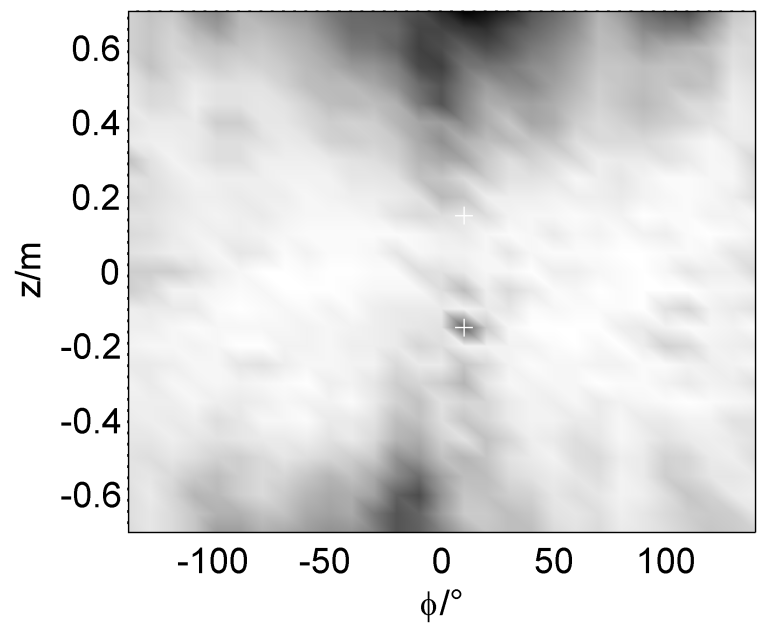

(b) Gray-scale map.

Figure 16. Sound source identification using focused beamforming.

\section{ACKNOWLEDGMENTS}

Supported by the National Natural Science Foundation of China (Grant No.11674075)

\section{REFERENCES}

1 Maynard J. D., Williams E. G. and Lee Y., Near field acoustic holography: I Theory of generalized holography and the development of NAH, J. Acoust. Soc. Am., 78 (4), 13951413, (1985). https://dx.doi.org/10.1121/1.392911

2 Williams E. G., Dardy H. D. and Washburn K. B., Generalized nearfield acoustical holography for cylindrical geometry: Theory and experiment, J. Acoust. Soc. Am., 110 (81), 389-407, (1987). https://dx.doi.org/10.1121/1.394904

3 Bai M. R. and Lin J. H., A new noise source identification technique: Nearfield acoustical beamformer (NABF), Proc. INTER-NOISE and NOISE-CON Congress and Conference, Honolulu (2006).

4 Yong T. C. and Roan M. J., Adaptive nearfield beamforming techniques for sound source imaging, J. Acoust. Soc. Am., 125 (2), 944-957, (2009). https://dx.doi.org/10.1121/1.3050248 


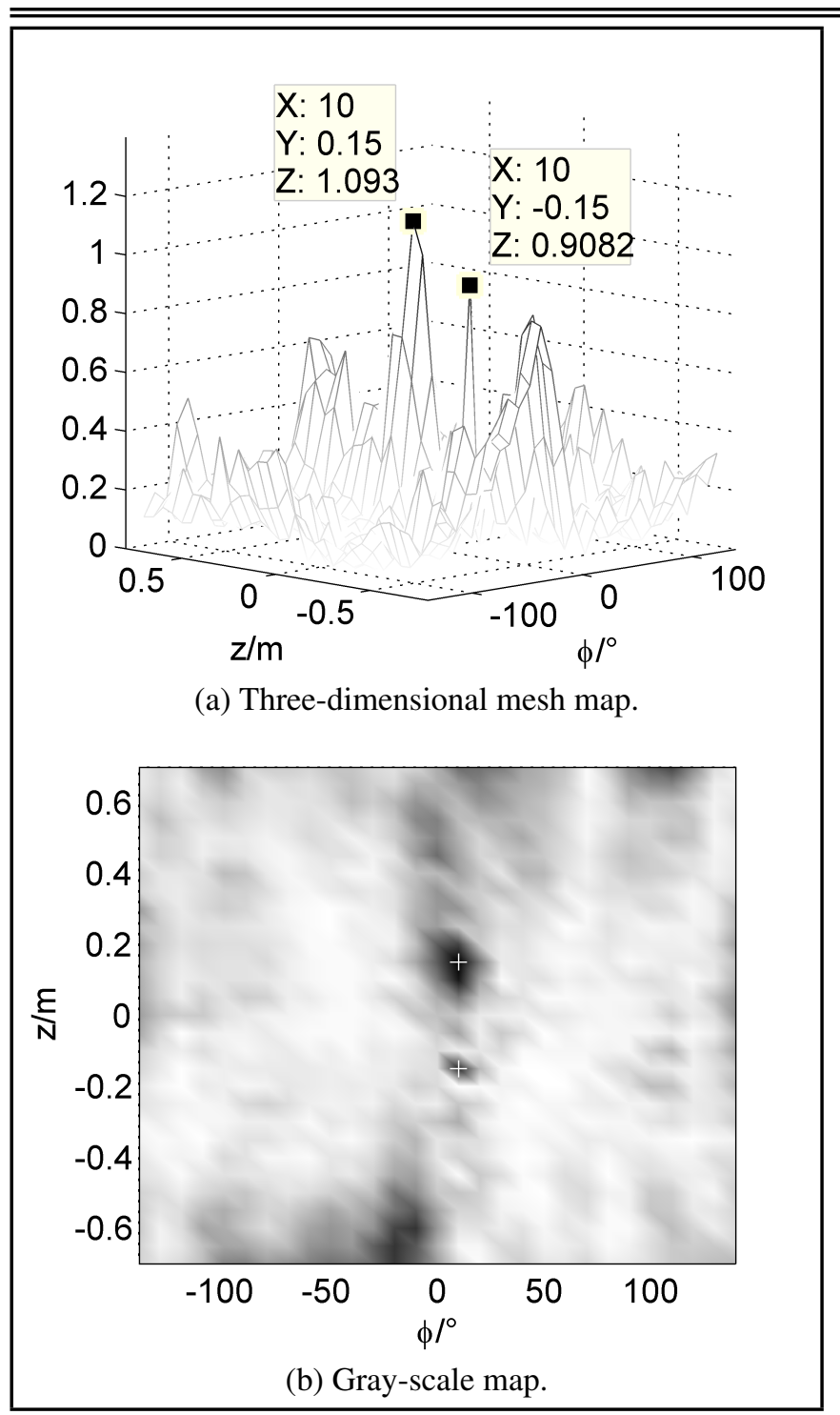

Figure 17. Sound source identification using the combined method.

5 Williams E. G., The beginnings of nearfield acoustical holography under Eugen Skudrzyk, J. Acoust. Soc. Am., 91 (4), 2335, (1992). https://dx.doi.org/10.1121/1.403501

6 Veronesi W. A. and Maynard J. D., Nearfield acoustic holography (NAH) II. Holographic reconstruction algorithms and computer implementation, J. Acoust. Soc. Am., 81 (5), 1307-1322, (1987). https://dx.doi.org/10.1121/1.394536

7 Villot M., Chavriat G. and Roland J., Phonoscopy: An acoustical holography technique for plane structures radiating in enclosed spaces, J. Acoust. Soc. Am., 91 (1), 187195, (1992). https://dx.doi.org/10.1121/1.402766

8 Hallman D., Bolton J. S. and Long L. B., The Application of Nearfield Acoustical Holography to Locate Sources in Enclosed Spaces Exhibiting Acoustic Modal Behavior, Proceedings of SPIE - The International Society for Optical Engineering (1994).

9 Pachner J., Investigation of Scalar Wave Fields by Means of Instantaneous Directivity Patterns, J. Acoust. Soc. Am., 28 (1), 90-92, (1956). https://dx.doi.org/10.1121/1.1908234
10 Weinreich G. and Arnold E. B., Method for measuring acoustic radiation fields, J. Acoust. Soc. Am., 68 (2), 404411, (1980). https://dx.doi.org/10.1121/1.384751

11 Hallman D. L. and Bolton J. S., Nearfield acoustical holography for interior sound fields, Proc Inter-Noise 94, Yokohama, Japan (1994).

12 Hallman D. and Bolton J. S., A technique for performing source identification in reflective environments by using nearfield acoustical holography, Proc. Noise-Con (1993).

13 Hallman D. L., Dumbacher S. M. and Libbey B. W., Acoustic Source Location in Vehicle Cabins and Free-field with Nearfield Acoustical Holography via Acoustic Arrays, Proc. the 19th International Seminar on Modal Analysis, Leuven, Belgium (1994).

14 Williams E. G., The nearfield acoustical holography (NAH) experimental method applied to vibration and radiation in light and heavy fluids, Computers and Structures, 65 (3), 323335, (1997). https://dx.doi.org/10.1016/s00457949(96)00253-2

15 Yuanan H. E., Rul L. I. and Zuoyong H. E., A technique of reconstructing reflectionmcoefficients at arbitrary angles of incidence, Acta Acustica (1996).

16 Williams E.G. and Houston B.H., Interior nearfield acoustical holography in flight, J. Acoust. Soc. Am., 108 (4), 14511463, (2000). https://dx.doi.org/10.1121/1.1289922

17 Yu F., Chen J. and Chen X.Z., Sound field separation technique with double holographic planes and its applications in acoustic holography, Chinese Journal of Acoustics, 28 (5), 385-389, (2003).

18 Yu F., Chen J. and Li W., Sound field separation technique and its applications in near-field acoustic holography, Journal of Physics, 54 (2), 789-797, (2005).

19 Hald J., Patch holography in cabin environments using a two-layer handheld array with an extended SONAH algorithm, Proc. Euronoise, Tampere (2006).

20 Melon M., Langrenne C., Rousseau D. and Herzog R., Comparison of Four Subwoofer Measurement Techniques, Journal of the Audio Engineering Society, 55 (12), 10771091, (2007).

21 Bi C. X., Chen X. Z. and Chen J., Sound field separation technique based on equivalent source method and its application in near field acoustic holography, J. Acoust. Soc. Am., 123 (3), 1472-1478, (2008). https://dx.doi.org/10.1121/1.2837489

22 Melon M., Langrenne C. and Herzog P., Evaluation of a method for the measurement of subwoofers in usual rooms, J. Acoust. Soc. Am., 127 (1), 256-263, (2010). https://dx.doi.org/10.1121/1.3270392

23 Pascal J. C. and Li J. F., On the use of double layer beamforming antenna for industrial applications, Proc. Euronoise, Naples, Italy (2003).

${ }^{24}$ Li Y. Z., Localization of Sound Sources by Beamforming, Dissertation for the master Degree, Hefei Polytechnic University (2012). 